\title{
Affective context interferes with cognitive control in unipolar depression: An fMRI investigation
}

\author{
Gabriel S. Dichtera,b,c,d, ${ }^{*}$, Jennifer N. Felder ${ }^{b}$, and Moria J. Smoski ${ }^{d}$ \\ aDepartment of Psychiatry, University of North Carolina at Chapel Hill School of Medicine, CB\# \\ 3366, Chapel Hill, NC 27599-7160, United States \\ bNeurodevelopmental Disorders Research Center, University of North Carolina at Chapel Hill \\ School of Medicine, CB\# 3366, Chapel Hill, NC 27599-7160, United States \\ 'Duke-UNC Brain Imaging and Analysis Center, Duke University Medical Center, Durham, NC, \\ 27710, United States \\ dDepartment of Psychiatry and Behavioral Sciences, Duke University Medical Center, Box 3026, \\ Durham NC 27710, United States
}

\begin{abstract}
Background-Unipolar major depressive disorder (MDD) is characterized by aberrant amygdala responses to sad stimuli and poor cognitive control, but the interactive effects of these impairments are poorly understood.
\end{abstract}

Aim-To evaluate brain activation in MDD in response to cognitive control stimuli embedded within sad and neutral contexts.

\begin{abstract}
Method-Fourteen adults with MDD and fifteen matched controls participated in a mixed block/ event-related functional magnetic resonance imaging (fMRI) task that presented oddball target stimuli embedded within blocks of sad or neutral images.
\end{abstract}

\begin{abstract}
Results-Target events activated similar prefrontal brain regions in both groups. However, responses to target events embedded within blocks of emotional images revealed a clear group dissociation. During neutral blocks, the control group demonstrated greater activation to targets in the midfrontal gyrus and anterior cingulate relative to the MDD group, replicating previous findings of prefrontal hypo-activation in MDD samples to cognitive control stimuli. However, during sad blocks, the MDD group demonstrated greater activation in a number of prefrontal regions, including the mid-, inferior, and orbito-frontal gyri and the anterior cingulate, suggesting that relatively more prefrontal brain activation was required to disengage from the sad images to respond to the target events.
\end{abstract}

\footnotetext{
(C) 2008 Elsevier B.V. All rights reserved.

*Corresponding author. Department of Psychiatry, University of North Carolina School of Medicine, CB\# 3366, Chapel Hill, NC 27599-3366, United States. Tel.: +1 919681 3169. E-mail address: dichter@med.unc.edu (G.S. Dichter).

This article appeared in a journal published by Elsevier. The attached copy is furnished to the author for internal non-commercial research and education use, including for instruction at the authors institution and sharing with colleagues.

Other uses, including reproduction and distribution, or selling or licensing copies, or posting to personal, institutional or third party websites are prohibited.

In most cases authors are permitted to post their version of the article (e.g. in Word or Tex form) to their personal website or institutional repository. Authors requiring further information regarding Elsevier's archiving and manuscript policies are encouraged to visit: http://www.elsevier.com/copyright

Conflict of interest

None of the authors have any commercial or financial conflicts of interest related to this work.
} 
Limitations-A larger sample size would have provided greater statistical power, and more standardized stimuli would have increased external validity.

Conclusions-This double dissociation of prefrontal responses to target events embedded within neutral and sad context suggests that MDD impacts not only responses to affective events, but extends to other cognitive processes carried out in the context of affective engagement. This implies that emotional reactivity to sad events in MDD may impact functioning more broadly than previously understood.

\section{Keywords}

Unipolar depression; Target detection; Cognitive control; Functional magnetic resonance imaging; Prefrontal cortex; Amygdala

\section{Introduction}

Emotions are mediated by complex neurobiological systems that prepare an organism to act appropriately in response to environmental stimuli and challenges (Gross, 1988). Emotional reactivity is mediated through limbic brain regions that have extensive dopaminergicallymediated inhibitory prefrontal modulatory projections (e.g., Jentsch et al., 2000; Ledoux, 2000). In non-clinical populations, responses to stimuli requiring cognitive control recruit dorsal prefrontal brain regions, including the anterior cingulate and the dorsolateral prefrontal cortex (Yamasaki et al., 2002; Fichtenholtz et al., 2004). Ventral brain regions, including the limbic system, and, in particular, the amygdala, respond to negatively valenced stimuli (Dolcos et al., 2006; Wang et al., 2005; Ledoux, 2007). Of relevance in the present context is the well established finding that limbic responses to affective stimuli interfere with prefrontallymediated, putatively non-affective cognitive processes (Dolcos et al., 2006; Dolcos and Mccarthy, 2006; Dolcos et al., 2007; Dolcos et al., 2008). Specifically, activity of ventral brain regions, including the amygdala as well as the ventrolateral and medial prefrontal cortex, inhibits functioning of dorsal "executive" brain regions (Seibert and Ellis, 1991), and dorsal functions are dampened when ventral systems are engaged (Dolcos and Mccarthy, 2006).

The purpose of the present investigation was to examine the effects of amygdala activity in response to sad stimuli on prefrontal responses to cognitive control stimuli in unipolar major depressive disorder (MDD). The core symptoms of MDD strongly implicate difficulties in emotion processing (i.e., sadness and anhedonia, American Psychiatric Association, 1994), and MDD is characterized by heightened reactions to sad stimuli, particularly after the termination of sad events, relative to individuals without MDD (Peeters et al., 2003; Rottenberg, 2005). Neuropsychological profiles of individuals with MDD reveal poor performance on tasks that measure cognitive control (Veiel, 1997; Zakzanis et al., 1998), deficits that have been linked to dysfunction of prefrontal brain regions, including the dorsolateral and ventrolateral prefrontal cortex and the anterior cingulate cortex (Brody et al., 2001; Rogers et al., 2004).

Individuals with MDD show greater amygdala activity during passive viewing of unpleasant stimuli (Surguladze et al., 2005), during encoding of subsequently remembered negative stimuli (Hamilton and Gotlib, 2008), and during automatic emotion processing (Dannlowski et al., 2007). Of relevance in the present context is evidence that MDD is characterized by more intense reactions to sad events after the termination of sad stimuli (Peeters et al., 2003; Goplerud and Depue, 1985), suggesting that the effects of amygdala hyperactivity to sad events may impact not only responses to sad stimuli per se, but on events that follow such stimuli as well. 
Several studies have made strides towards demonstrating the effects of emotional hyperresponsivity on cognitive processing in MDD. Siegle et al. (2007) reported amygdala hyperactivity to personally relevant negative words and dorsolateral prefrontal hypoactivity to a cognitive task (i.e., digit sorting); however the two tasks were presented in relative isolation (see also Johnstone et al., 2007). Likewise, Wang et al. (2008) demonstrated the impact of emotional stimuli on subsequent hemodynamic responses to a cognitive control task in MDD, but the study examined delayed effects of emotional stimuli (18-20 s after offset), and medication use by MDD participants appeared to blunt neural reactivity to the emotional stimuli. Grimm et al. (2008) reported anomalous DLPFC activity during emotional judgments of International Affective Picture System (Lang et al., 2005) images. Finally, Fales et al. (2008) reported anomalous relations between activity of the amygdala and the prefrontal cortex in response to unattended fear images during an attentional interference task. However, the use of fearful (as opposed to sad or MDD-specific) stimuli is less diagnostically relevant to MDD, and there are inconsistent findings of cognitive bias to fear-related negative stimuli in MDD (Mogg and Bradley, 2005).

These converging lines of evidence suggest that MDD is characterized by aberrant limbicprefrontal interactions. However, the effects of amygdala hyper-reactivity on prefrontal recruitment to cognitive control stimuli presented in the context of sad stimuli in MDD are unknown. This is a critical omission, given that the impact of poorly modulated emotional responses in MDD likely extends beyond responses to affective events themselves to other domains of cognitive processes that are essential for effective functioning. Attempts to perform tasks requiring cognitive control during a sad context likely mirror the real-world experience of individuals with MDD and highlights the impact of affective events on other domains of cognitive processing that are required for productive functioning.

The purpose of this investigation was to examine limbic-prefrontal interactions in unmedicated adults with MDD via a mixed block/event target detection task using functional magnetic resonance imaging (fMRI). This design allows for analyses of responses to cognitive control stimuli presented during blocks of neutral and sad images. When target events were presented within blocks of neutral images, we predicted that we would replicate previous studies documenting prefrontal hypo-activation in MDD during tasks requiring cognitive control (e.g., Brody et al., 2001; Rogers et al., 2004). However, we hypothesized a different pattern of results for target events embedded within blocks of sad images: specifically, we hypothesized that successful target detection during blocks of sad images would require MDD participants to recruit prefrontal regions to a greater degree than their nondepressed counterparts. We hypothesized increased prefrontal activation to cognitive control stimuli in sad contexts in the MDD group because of evidence that the prefrontal cortex mediates affect regulation in emotional contexts (Ochsner et al., 2004) and that intact behavioral performance in the context of increased task demands predicts greater prefrontal neuronal activity that acts in a compensatory manner (Adler et al., 2001). In other words, we predicted that hyper-responsivity to sad images would require greater "cognitive effort" to respond appropriately to cognitive control stimuli (e.g., Matsuo et al., 2007).

We further hypothesized that greater severity of depressive symptoms would predict increased "cognitive effort" to disengage from sad stimuli, and thus greater prefrontal activation to targets embedded within sad blocks. We hypothesized no group differences on subjective responses to affective images, based on published findings (e.g., Dichter et al., 2004; Dichter and

\footnotetext{
${ }^{1}$ On the basis of mostly self-report data, Rottenberg et al. $(2005,2007)$ have argued that depression is associated with broad emotioncontext insensitivity, suggesting hypo-responsivity to both pleasant and unpleasant events. We note that in the present study, we are examining specifically amygdala responses to sad stimuli, rather than the effects of sad stimuli on self-reported mood.
} 
Tomarken, 2008; but see, e.g., Sloan et al., 1997; Sloan et al., 2001; Rottenberg et al., 2005), and no group differences in task accuracy and reaction times, given the ease of the task.

\section{Materials and methods}

\subsection{Participants}

Sixteen unmedicated adults with unipolar major depressive disorder (MDD) and 15 control participants enrolled in the study. One depressed female participant withdrew after her diagnostic interview due to remission of symptoms. Not included in analyses are the data from one depressed female who had frank abnormalities in brain anatomy. The final sample included fourteen participants with MDD (7 female; 3 African American, 1 Asian American, 2 Hispanic ethnicity, 1 "Other") and 15 control adults with no current Axis I disorders and no history of MDD (9 female; 2 "Other"). One MDD participant met criteria for concurrent dysthymia. Participants were recruited via local newspaper and web advertisements, as well as flyers posted in campus and medical center locations. After a complete description of the study was provided to participants, written informed consent was obtained to protocols approved by the Duke and UNC-CH Human Investigations Committees.

Participants were assessed using the Structured Clinical Interview for DSM-IV Axis I Disorders (SCID-I; First et al., 1995) and the North American Adult Reading Test (NAART, Blair and Spreen, 1989). Inclusion criteria for the MDD group included the presence of current Major Depressive Disorder and no other current Axis I disorder other than dysthymia. Inclusion criteria for the control group included no current Axis I disorder and no history of mood disorder. Exclusion criteria for both groups included current use of psychotropic medications, estimated IQ of 80 or lower, history of neurological disorders or injury, current substance abuse or dependence, or factors that would interfere with the safety of MRI scanning (e.g., implanted metal, claustrophobia, current pregnancy).

Participants were paid $\$ 10$ for each hour of diagnostic and symptom assessments, and $\$ 45$ for each imaging session. The depressed (mean=26.9, $\mathrm{SD}=4.9$ ) and nondepressed (mean=0.7, $\mathrm{SD}=1.2$ ) groups differed significantly with respect to Beck Depression Inventory (BDI, Beck et al., 1996) scores, $t(27)=20.6, p<.0001$, but not with respect to age, $t(27)=1.99, p>.05$ [MDD mean $(\mathrm{SD})$ age $=34.8$ (14.3) years, control mean $(\mathrm{SD})=30.8(9.6)$ years], or gender distribution, $\chi^{2}(1)=0.3, p>.10$.

\section{2. fMRI task}

Participants completed seven functional imaging runs (see Fig. 1). Runs 1-5 each consisted of a 5'38" forced-choice mixed block and event-related target detection task during which a rare target stimulus (i.e., a bullseye) was presented embedded within alternating blocks of sad and neutral pictures. Runs began and ended with a neutral block. Stimuli were presented for 1000 $\mathrm{ms}$ and with a $2000 \mathrm{~ms}$ stimulus onset asynchrony (SOA). Blocks were $30 \mathrm{~s}$ in duration and consisted of two target stimuli embedded within 13 non-target emotional pictures. All target events were separated by a minimum of $12 \mathrm{~s}$, and targets were not presented within the first 6 $\mathrm{s}$ of each block. In this forced-choice reaction time paradigm, participants were instructed to respond via right-hand button box to every stimulus as quickly and accurately as possible by pressing one button for all non-target images and an alternate button for targets. In this manner, motor activity related to making button-presses was incorporated into the task baseline.

Runs 6 and 7 were identical to runs 1-5, however target events were not presented and button responses were not required (i.e., a passive viewing block design task). The purpose of these runs was to validate whether the sad images, not presented in the context of a target detection task, recruited the amygdala to a greater degree in the MDD sample. This design feature was 
incorporated to allow for testing of whether the MDD group did indeed evidence hyperactivity to sad stimuli without the potential confounds of (1) embedded target events in the mixed block/ event runs, and (2) the executive demands of button-presses to sad stimuli in the mixed block/ event runs.

\subsection{Stimuli}

Visual stimuli were identical to those developed by Wang et al. (2005) specifically for studies of MDD. Given that pervasive sadness is a diagnostic feature of MDD, images were chosen to elicit that particular emotion. An insufficient number of images from the more commonly used International Affective Picture System (Lang et al., 2005) elicit the specific emotion of sadness (Mikels et al., 2005), and thus we employed an image set that was designed and previously normed to assess responses to sad stimuli (Wang et al., 2005). In a subsequent study by Wang and colleagues, both controls and MDD participants again rated the images as sad, with MDD participants more likely to rate the images as "very sad" than controls (Wang et al., 2008).

This grayscale stimulus set contains 56 sad and 54 neutral images. Sad images were those that elicited average sadness ratings of 2 or higher on a 3-point sadness intensity scale ( $1=$ not sad/ unsure, $2=$ mildly sad, $3=\mathrm{sad}$ ) from a non-clinical validation sample. The sad pictures contained scenes of humans crying or portrayed sad facial expressions. The neutral images were matched as closely as possible to the final pool of sad pictures for presence and number of human figures in the image, postural features, gaze direction, and gender. The initial fMRI validation study employing these images confirmed robust amygdala activation in response to these sad images (Wang et al., 2005). Stimuli were presented using CIGAL presentation software (Voyvodic, 1999) and displayed to the participants through magnet-compatible goggles (Resonance Technology, Inc., Northridge CA).

After the fMRI session was complete, pictures were presented again, outside of the scanner, and participants rated each with respect to pleasure and arousal using the Self Assessment Manikin (Bradley and Lang, 1994), a 9-point Likert pictorial assessment technique.

\subsection{Imaging}

Scanning was performed on a General Electric 4T LX NVi MRI scanner system equipped with $41 \mathrm{mT} / \mathrm{m}$ gradients (General Electric, Waukesha, Wisconsin, USA). A quadrature birdcage radio frequency $(\mathrm{RF})$ head coil was used for transmit and receive. The participant's head was immobilized using blocks of foam. Sixty-eight high resolution images were acquired using a 3D fast SPGR pulse sequence $(\mathrm{TR}=12 \mathrm{~ms} ; \mathrm{TE}=5.4 \mathrm{~ms} ; \mathrm{FOV}=24 \mathrm{~cm} ;$ image matrix $=256$ $\times 192$; voxel size $=0.9375 \times 0.9375 \times 1.9 \mathrm{~mm} ; 72$ oblique axial slices; $\delta=20^{\circ}$ ) and used for coregistration with the functional data. Structural images were aligned in a near axial plane defined by the anterior and posterior commissures. Whole brain functional images were acquired using an echoplanar pulse sequence sensitive to blood oxygenation level dependent (BOLD) contrast (TR, $2000 \mathrm{~ms}$; TE, $25 \mathrm{~ms}$; FOV, $24 \mathrm{~cm}$; image matrix $=64^{2}$; $\delta=60^{\circ}$; voxel size, $3.75 \times 3.75 \times 3.8 \mathrm{~mm} ; 34$ axial slices). The functional images were aligned similarly to the structural images. A semi-automated high-order shimming program ensured global field homogeneity.

\subsection{Imaging data analysis}

Head motion was analyzed by center of mass measurements in three orthogonal planes, and imaging epochs with mean intensities greater than three standard deviations of the average intensity in a run were excluded from analyses. During runs $1-5$, only epochs during which participants gave a correct response were included in analyses. 
Functional data were preprocessed using FSL version 4.0.2 (Oxford Centre for Functional Magnetic Resonance Imaging of the Brain (FMRIB), Oxford University, U.K.). Timing files were converted to FSL compatible format and NIFTI image data files were generated. Preprocessing was applied in the following steps: (i) brain extraction for non-brain removal (Smith et al., 2004), (ii) motion correction using MCFLIRT (Smith, 2002), (iii) spatial smoothing using a Gaussian kernel of FWHM $5 \mathrm{~mm}$, (iv) mean-based intensity normalization of all volumes by the same factor, and (v) high-pass filtering (Jenkinson et al., 2002). Functional images of each subject were co-registered to structural images in native space, and structural images were normalized into a standard stereotaxic space (Montreal Neurological Institute) for intersubject comparison. The same transformation matrices used for structural-to-standard transformations were then used for functional-to-standard space transformations of coregistered functional images. All registrations were carried out using an intermodal registration tool (Jenkinson et al., 2002; Smith et al., 2004). Voxel-wise temporal autocorrelation was estimated and corrected using FMRIB's Improved Linear Model (Jenkinson and Smith, 2001).

Onset times of events were used to model a signal response containing a regressor for each response type, which was convolved with a double- $\gamma$ function to model the hemodynamic response. Model fitting generated whole brain images of parameter estimates and variances, representing average signal change from baseline (activation; positive regressor) and below baseline (deactivation; negative regressor). Group-wise activation and deactivation images were calculated by amixed effects higher level analysis using Bayesian estimation techniques, FMRIB Local Analysis of Mixed Effects (FILM, Woolrich et al., 2001) with a conservative cluster mean threshold of $Z>2.3$ and a cluster-corrected significance threshold of $p<0.05$ (FLAME 1 + 2) (Beckmann et al., 2003).

\section{Results}

\subsection{Imaging data}

Fig. 2 illustrates within-group activation patterns to all target events, collapsed across sad and neutral blocks. The figure illustrates that in both diagnostic groups, target events activated a common prefrontal network, including the anterior and posterior cingulate gyrus, the midfrontal gyrus, and the inferior frontal gyrus. Strikingly, there were no statistically significant group differences with respect to prefrontal activation patterns in response to target events, collapsed across sad and neutral blocks (data not shown).

Fig. 3 illustrates between-groups activation pattern differences with respect to target events embedded within blocks of emotional images. Depicted are areas of greater activation in the control group to targets presented within neutral blocks (in red) and areas of greater activation in the MDD group to targets presented within sad blocks (in blue). The figure illustrates that the only prefrontal region characterized by relatively greater control group activation (in red) to targets embedded within neutral blocks was the left midfrontal gyrus. The MDD group demonstrated no prefrontal areas with greater activation to this contrast (data not shown). However, the MDD group demonstrated relatively greater activation to target events embedded within sad blocks (in blue) in the mid-, inferior, and orbito-frontal gyrus and the anterior cingulate. There were no prefrontal lobe areas with greater activation to target events embedded within sad blocks in the control group relative to the MDD group, and there were no clusters with greater activation in the control group relative to the MDD group during targets within sad blocks (data not shown). Table 1 indicates activation coordinates for these contrasts.

The top of Fig. 4 depicts areas of greater activation to sad blocks from the block-only runs (i.e., runs 6 and 7), and illustrates that the MDD group demonstrated relatively greater activation in the left amygdala to sad images than the control group. 
Finally, we assessed for relations between regional brain activation values and depressive symptom severity in an exploratory fashion. The bottom of Fig. 4 depicts results of covariate analyses illustrating a significant correlation between bilateral midfrontal activations in response to target events embedded within sad blocks and Beck Depression Inventory (BDI) scores.

\subsection{Behavioral performance}

The top of Fig. 5 illustrates accuracy and latency in response to targets embedded within blocks of sad and neutral pictures. An omnibus 2 (Group: depressed, nondepressed) $\times 2$ (Condition: sad targets, neutral targets) repeated-measures MANOVA on accuracy data revealed no main effect of Condition or Group $\times$ Condition interaction, $p$ ' $s>.15$, but a trend towards a main effect of Group, $F(1,27)=2.98, p<0.10$. Within both diagnostic groups alone, there were no accuracy differences between responses to conditions, $p$ 's $<.25$. A similar analysis of latency data revealed no main effect of Condition, Group, or Group $\times$ Condition interaction, $p$ ' $s<.30$. Within both diagnostic groups alone, there were no latency differences between responses to picture categories, $p$ 's $<.85$.

\subsection{Self-report responses to pictures}

The bottom of Fig. 5 depicts mean picture ratings for both diagnostic groups. A Group (depressed, nondepressed) $\times$ Valence (neutral, sad) ANOVA on pleasure ratings revealed a main effect of Valence, multivariate $F(1,27)=217.61, p<0.0001$, indicating that, for both groups, valence ratings followed the pattern of the a priori valence categories (i.e., sad pictures were rated as less pleasant than neutral pictures), but no main effect of Group or Group $\times$ Valence interaction, $p$ 's $>0.35$. Follow-up between-groups $t$ tests revealed no group differences in valence ratings of either picture category, $p$ 's $>0.40$. A similar analysis of arousal ratings revealed a main effect of Valence, multivariate $F(1,27)=32.05, p<0.0001$, indicating that both groups found the sad images to be more arousing than the neutral images, but no main effect of Group or Group $\times$ Valence interaction, $p$ 's $>0.20$. Follow-up between-groups $t$ tests revealed no group differences in arousal ratings of either picture category, $p$ 's $>0.25$.

\section{Discussion}

The purpose of the present investigation was to examine the influence of limbic hyperreactivity to sad events on functioning of prefrontal brain regions in response to cognitive control stimuli in MDD via a mixed block/event target detection task. The oddball task presented rare target events embedded within blocks of neutral and sad images, thereby allowing for separate examinations of prefrontal recruitment in response to target events embedded within sad and neutral blocks. Affective images were employed that have been shown to elicit sadness specifically and to recruit amygdala activation in control and MDD samples (Wang et al., 2005, 2008).

Analyses focused on responses to target events, both overall and within sad and neutral blocks separately. Analyses of responses to all target events, regardless of whether they were embedded within sad or neutral blocks, did not reveal evidence of prefrontal hypo-activation in the MDD group in response to the cognitive control task. However, group differences emerged when analyses were constrained to responses to target events embedded within either the sad or neutral blocks.

Analyses of responses to cognitive control stimuli embedded within neutral blocks revealed relatively greater midfrontal and anterior cingulate activation in the control group. This pattern of findings is broadly consistent with published findings of prefrontal hypo-activation in MDD during cognitive control tasks (Brody et al., 2001; Rogers et al., 2004; Robertson et al., 
2007). However, analyses of responses to cognitive control stimuli embedded within sad blocks revealed relatively greater prefrontal activation in the MDD group. This is the first report, to our knowledge, of relatively greater prefrontal activation in MDD to cognitive control stimuli presented within a sad context. These results suggest that prefrontal dysfunction in MDD may take a different form in sad contexts than in neutral or baseline contexts. Responses to passive viewing block-only runs revealed greater left amygdala responses to sad stimuli in the MDD group, providing support for the conclusion that amygdala hyper-activation in the MDD group to sad images resulted in relatively greater prefrontal recruitment to target stimuli. Because analyses were restricted to trials with correct behavioral responses (i.e., correct button responses to target events), these group differences reflect differential neural recruitment by emotional block conditions rather than behavioral performance differences.

The prefrontal cortex is known to mediate a number of goal-directed behaviors. Relevant in the present context is evidence that the midfrontal and inferior frontal gyrus and the anterior cingulate mediate cognitive control and cognitive interference (Bunge et al., 2001; Fan et al., 2005; Fichtenholtz et al., 2004). We conclude that the pattern of increased prefrontal activation in this context in the MDD group reflected that greater "cognitive effort" was required (e.g., Matsuo et al., 2007) to disengage from the sad images to respond to target events. In other words, it may be the case that responding to target events embedded within sad blocks required, in essence, greater cognitive control in the MDD group because of greater engagement with the task-irrelevant images.

Exploratory covariate analyses of relations between brain activations to target events embedded within sad blocks and the severity of depressive symptoms revealed a direct association between activity of bilateral midfrontal cortex and BDI scores. Consistent with the hypothesis that greater severity of depression symptoms would require greater "cognitive effort" to disengage from sad stimuli, we found that $70 \%$ of the variance of activation values was explained by BDI scores. Clearly, this finding merits replication in larger samples, but suggests that depression severity is directly linked to the effects of affective interference on cognitive control in unipolar MDD.

We note that that in addition to our relatively small sample size, an added interpretive caveat of this study is the particular images used to induce sadness. Though the image set has been employed in other studies of affective processing in MDD and control participants (Wang et al., 2005, 2008), with both control and MDD groups rating the images as inducing sadness, it is relatively less well normed than other stimulus sets designed to induce emotional responses (e.g., Lang et al., 2005). Though the present study presents confirmatory self-report evidence that negative emotion was induced by the images in both groups (i.e., ratings of valence were lower to sad images than neutral images in both groups), specific ratings of sadness were not collected. Additionally, the image set used in the present study was designed to induce sadness but not necessarily high levels of physiological arousal. Although this stimulus set has been demonstrated to recruit amygdala activation in prior clinical and non-clinical studies (Wang et al., 2005, 2008), high levels of induced arousal are known to enhance amygdala activation (Dalton et al., 2005). Thus, it is possible that amygdala responses were attenuated by the affective qualities of the stimuli employed. Further research using the present stimuli will allow for comparisons with other affective picture sets. We also note that evidence of amygdala hyper-activation to sad images during the mixed block/event runs (i.e., runs 1-5) was drawn from data collected during passive viewing block runs (runs 6 and 7). As described earlier, this design feature was chosen because of the possibly confounding effects of analyzing responses to sad event embeddedwithin the target detection task that required behavioral responses.

Analyses of in-scanner accuracy and reaction times did not reveal group differences, nor did self-report valence and arousal ratings of the images. The disparity between fMRI and 
behavioral data may be due to the relative ease of the target detection task, which resulted in MDD participants demonstrating no differences in behavioral responses, despite clear disparities in fMRI data. We thus conclude that brain imaging data provided unique information not discernable from behavioral or self-report measures. Though current models of emotion regulation (e.g., Taylor and Liberzon, 2007) suggest a decreased ability to exert cognitive control in MDD that would result in more behavioral interference and less prefrontal activity, the fMRI task in the present study was simple enough that groups did not differ in behavior. Thus the present findings should be viewed as reflective of functional prefrontal characteristics in MDD in the context of intact behavioral performance. ${ }^{2}$ In this regard, we interpret our primary findings to indicate that increased prefrontal activity in sad contexts in the MDD group reflects a compensatory process, as evidenced by intact behavioral performance, relative to nondepressed participants.

In summary, this is the first report to our knowledge of the effects of immediate sad context on the neural correlates of cognitive control in MDD. These results represent fMRI evidence that prolonged or enhanced processing of sad events impacts environmental responding even after the termination of the sad stimulus, and are consistent with self-report data that MDD is characterized by enhanced reactions to sad stimuli after the termination of sad events (Peeters et al., 2003; Rottenberg, 2005). The differential effect of sad versus neutral context on prefrontal activation during cognitive control, with hyper-activation in the former and hypoactivation in the latter, also highlights the critical importance of current mood state and affective context on cognition in MDD. We conclude that future studies of cognitive control deficits in MDD should assess the boundary conditions of anomalous prefrontal responses to cognitive control tasks.

\section{Acknowledgments}

The authors would like to thank Josh Bizzell, Chris Petty, Todd Harshbarger, and Syam Gadde for assistance with image analysis, Justin Woodlief, Shian Ling Keng, and Prue Cuper for assistance with data collection, and MRI technologists Susan Music, Natalie Goutkin, and Talaignair Venkatraman for assistance with data acquisition. We thank Drs. Aysenil Belger, Thomas Lynch, and Lihong Wang for assistance with various aspects of this research. We thank two anonymous reviewers for their helpful comments on an earlier version of this manuscript.

Role of funding source

This investigation was supported by grants from the National Institute of Mental Health (MH078145) and the National Alliance for Research in Schizophrenia and Affective Disorders (NARSAD) to G. Dichter. Assistance for this study was provided by the Neuroimaging Core of the UNC Neurodevelopmental Disorders Research Center. G. Dichter was supported by Postdoctoral Research in Neurodevelopmental Disorders, NICHD T32-HD40127 and a career development award from UNC-Chapel Hill, NIH/NCRR K12 RR023248. M. Smoski was supported by NIMH T32MH070448. The funding sources had no further roles in study design, in the collection, analysis, and interpretation of data; in the writing of the report; and in the decisions to submit the paper for publication.

\section{References}

Adler CM, Sax KW, Holland SK, Schmithorst V, Rosenberg L, Strakowski SM. Changes in neuronal activation with increasing attention demand in healthy volunteers: an fMRI study. Synapse 2001;42:266-272. [PubMed: 11746725]

American Psychiatric Association. Diagnostic and Statistical Manual of Mental Disorders. Vol. fourth edition. Washington, DC: DSM-IV; 1994.

Beck, AT.; Steer, RA.; Brown, GK. Manual for Beck Depression Inventory-II. San Antonio, TX: Psychological Corporation; 1996.

Beckmann CF, Jenkinson M, Smith SM. General multilevel linear modeling for group analysis in FMRI. Neuroimage 2003;20:1052-1063. [PubMed: 14568475]

\footnotetext{
${ }^{2}$ We thank an anonymous review for suggesting this point.
} 
Blair JR, Spreen O. Predicting premorbid IQ: a revision of the national adult reading test. Clin. Neuropsychol 1989;3:129-136.

Bradley MM, Lang PJ. Measuring emotion: The Self-Assessment Manikin and the semantic differential. J. Behav. Ther. Exp. Psychiatry 1994;25:49-59. [PubMed: 7962581]

Brody AL, Barsom MW, Bota RG, Saxena S. Prefrontal-subcortical and limbic circuit mediation of major depressive disorder. Semin. Clin. Neuropsychiatry 2001;6:102-112. [PubMed: 11296310]

Bunge SA, Ochsner KN, Desmond JE, Glover GH, Gabrieli JD. Prefrontal regions involved in keeping information in and out of mind. Brain 2001;124:2074-2086. [PubMed: 11571223]

Dalton KM, Kalin NH, Grist TM, Davidson RJ. Neural-cardiac coupling in threat-evoked anxiety. J Cogn Neurosci 2005;17:969-980. [PubMed: 15969913]

Dannlowski U, Ohrmann P, Bauer J, Kugel H, Arolt V, Heindel W, Kersting A, Baune BT, Suslow T. Amygdala reactivity to masked negative faces is associated with automatic judgmental bias in major depression: a 3 T fMRI study. J. Psychiatry Neurosci 2007;32:423-429. [PubMed: 18043766]

Dichter GS, Tomarken AJ. The chronometry of affective startle modulation in unipolar depression. J. Abnorm. Psychology 2008;117:1-15.

Dichter GS, Tomarken AJ, Shelton RC, Sutton SK. Early- and late-onset startle modulation in unipolar depression. Psychophysiology 2004;41:433-440. [PubMed: 15102129]

Dolcos F, Mccarthy G. Brain systems mediating cognitive interference by emotional distraction. J. Neurosci 2006;26:2072-2079. [PubMed: 16481440]

Dolcos F, Kragel P, Wang L, Mccarthy G. Role of the inferior frontal cortex in coping with distracting emotions. Neuroreport 2006;17:1591-1594. [PubMed: 17001274]

Dolcos F, Miller B, Kragel P, Jha A, Mccarthy G. Regional brain differences in the effect of distraction during the delay interval of a working memory task. Brain Res 2007;1152:171-181. [PubMed: 17459348]

Dolcos F, Diaz-Granados P, Wang L, Mccarthy G. Opposing influences of emotional and non-emotional distracters upon sustained prefrontal cortex activity during a delayed-response working memory task. Neuropsychologia 2008;46:326-335. [PubMed: 17765933]

Fales CL, Barch DM, Rundle MM, Mintun MA, Snyder AZ, Cohen JD, Mathews J, Sheline YI. Altered emotional interference processing in affective and cognitive-control brain circuitry in major depression. Biol. Psychiatry 2008;63:377-384. [PubMed: 17719567]

Fan J, Mccandliss BD, Fossella J, Flombaum JI, Posner MI. The activation of attentional networks. Neuroimage 2005;26:471-479. [PubMed: 15907304]

Fichtenholtz HM, Dean HL, Dillon DG, Yamasaki H, Mccarthy G, Labar KS. Emotion-attention network interactions during a visual oddball task. Brain Res. Cogn. Brain Res 2004;20:67-80. [PubMed: 15130591]

First, MB.; Gibbon, M.; Spitzer, RL.; Williams, JBW. Structured Clinical Interview for DSM-IV Axis I Disorders, Research Edition (SCID-I/NP, Version 2.0). Washington, D.C: American Psychiatric Press, Inc.; 1995.

Goplerud E, Depue RA. Behavioral response to naturally occurring stress in cyclothymia and dysthymia. J. Abnorm. Psychology 1985 May;94(2):128-139.

Grimm S, Beck J, Schuepbach D, Hell D, Boesiger P, Bermpohl F, Niehaus L, Boeker H, Northoff G. Imbalance between left and right dorsolateral prefrontal cortex in major depression is linked to negative emotional judgment: an fMRI study in severe major depressive disorder. Biol. Psychiatry 2008;63:369-376. [PubMed: 17888408]

Gross JJ. The emerging field of emotion regulation: an integrative review. Rev. Gen. Psychol 1988;2:271299.

Hamilton JP, Gotlib IH. Neural substrates of increased memory sensitivity for negative stimuli in major depression. Biol. Psychiatry. 2008

Jenkinson M, Smith S. A global optimisation method for robust affine registration of brain images. Med. Image Anal 2001;5:143-156. [PubMed: 11516708]

Jenkinson M, Bannister P, Brady M, Smith S. Improved optimization for the robust and accurate linear registration and motion correction of brain images. Neuroimage 2002;17:825-841. [PubMed: 12377157] 
Jentsch JD, Roth RH, Taylor JR. Role for dopamine in the behavioral functions of the prefrontal corticostriatal system: implications for mental disorders and psychotropic drug action. Prog. Brain Res 2000;126:433-453. [PubMed: 11105661]

Johnstone T, Van Reekum CM, Urry HL, Kalin NH, Davidson RJ. Failure to regulate: counterproductive recruitment of top-down prefrontal-subcortical circuitry in major depression. J. Neurosci 2007;27:8877-8884. [PubMed: 17699669]

Lang, PJ.; Bradley, MM.; Cuthbert, BN. Technical Report A-6. Gainesville, FL: University of Florida; 2005. International affective picture system (IAPS): Digitized photographs, instruction manual and affective ratings.

Ledoux JE. Emotion circuits in the brain. Annu. Rev. Neurosci 2000;23:155-184. [PubMed: 10845062]

Ledoux J. The amygdala. Curr. Biol 2007;17:R868-R874. [PubMed: 17956742]

Matsuo K, Glahn DC, Peluso MA, Hatch JP, Monkul ES, Najt P, Sanches M, Zamarripa F, Li J, Lancaster JL, Fox PT, Gao JH, Soares JC. Prefrontal hyperactivation during working memory task in untreated individuals with major depressive disorder. Mol. Psychiatry 2007;12:158-166. [PubMed: 16983390]

Mikels JA, Fredrickson BL, Larkin GR, Lindberg CM, Maglio SJ, Reuter-Lorenz PA. Emotional category data on images from the International Affective Picture System. Behav. Res. Meth 2005;37:626630.

Mogg K, Bradley BP. Attentional bias in generalized anxiety disorder versus depressive disorder. Cogn. Ther. Res 2005;29:29-45.

Ochsner KN, Ray RD, Cooper JC, Robertson ER, Chopra S, Gabrieli JD, Gross JJ. For better or for worse: neural systems supporting the cognitive down- and up-regulation of negative emotion. Neuroimage 2004;23:483-499. [PubMed: 15488398]

Peeters F, Nicolson NA, Berkhof J, Delespaul P, Devries M. Effects of daily events on mood states in major depressive disorder. J. Abnorm. Psychology 2003;112:203-211.

Robertson B, Wang L, Diaz MT, Aiello M, Gersing K, Beyer J, Mukundan S Jr, Mccarthy G, Doraiswamy PM. Effect of bupropion extended release on negative emotion processing in major depressive disorder: a pilot functional magnetic resonance imaging study. J. Clin. Psychiatry 2007;68:261-267. [PubMed: 17335325]

Rogers MA, Kasai K, Koji M, Fukuda R, Iwanami A, Nakagome K, Fukuda M, Kato N. Executive and prefrontal dysfunction in unipolar depression: a review of neuropsychological and imaging evidence. Neurosci. Res 2004;50:1-11. [PubMed: 15288493]

Rottenberg J. Mood and emotion in major depression. Curr. Dir. Psychol. Sci 2005;14:167-170.

Rottenberg, J. Major depressive disorder: emerging evidence for emotion context insensitivity. In: Rottenberg, J.; Johnson, SL., editors. Emotion and Psychopathology: Bridging Affective and Clinical Science. Washington DC: American Psychological Society; 2007.

Rottenberg J, Gross JJ, Gotlib IH. Emotion context insensitivity in major depressive disorder. J. Abnorm. Psychology 2005;114:627-639.

Seibert PS, Ellis HC. Irrelevant thoughts, emotional mood states, and cognitive task performance. Mem. Cogn 1991;19:507-513.

Siegle GJ, Thompson W, Carter CS, Steinhauer SR, Thase ME. Increased amygdala and decreased dorsolateral prefrontal BOLD responses in unipolar depression: related and independent features. Biol. Psychiatry 2007;61:198-209. [PubMed: 17027931]

Sloan DM, Strauss ME, Quirk SW, Sajatovic M. Subjective and expressive emotional responses in depression. Journal of Affective Disorders 1997;46:135-141. [PubMed: 9479617]

Sloan DM, Strauss ME, Wisner KL. Diminished response to pleasant stimuli by depressed women. J. Abnorm. Psychology 2001;110:488-493.

Smith SM. Fast robust automated brain extraction. Hum. Brain Mapp 2002;17:143-155. [PubMed: 12391568]

Smith SM, Jenkinson M, Woolrich MW, Beckmann CF, Behrens TEJ, Johansen-Berg H, Bannister PR, De Luca M, Drobnjak I, Flitney DE, Niazy RK, Saunders J, Vickers J, Zhang Y, De Stefano N, Brady JM, Matthews PM. Advances in functional and structural MR image analysis and implementation as FSL. Neuroimage 2004;23:S208-S219. [PubMed: 15501092] 
Surguladze S, Brammer MJ, Keedwell P, Giampietro V, Young AW, Travis MJ, Williams SC, Phillips ML. A differential pattern of neural response toward sad versus happy facial expressions in major depressive disorder. Biol. Psychiatry 2005;57:201-209. [PubMed: 15691520]

Taylor SF, Liberzon I. Neural correlates of emotion regulation in psychopathology. Trends Cogn. Sci 2007;11:413-418. [PubMed: 17928261]

Veiel HO. A preliminary profile of neuropsychological deficits associated with major depression. J. Clin. Exp. Neuropsychol 1997;19:587-603. [PubMed: 9342691]

Voyvodic JT. Real-time fMRI paradigm control, physiology, and behavior combined with near real-time statistical analysis. Neuroimage 1999;10:91-106. [PubMed: 10417244]

Wang L, Mccarthy G, Song AW, Labar KS. Amygdala activation to sad pictures during high-field (4 tesla) functional magnetic resonance imaging. Emotion 2005;5:12-22. [PubMed: 15755216]

Wang L, Labar KS, Smoski M, Rosenthal MZ, Dolcos F, Lynch TR, Krishnan RR, Mccarthy G. Prefrontal mechanisms for executive control over emotional distraction are altered in major depression. Psychiatry Res 2008;163:143-155. [PubMed: 18455373]

Woolrich MW, Ripley BD, Brady M, Smith SM. Temporal autocorrelation in univariate linear modeling of FMRI data. Neuroimage 2001;14:1370-1386. [PubMed: 11707093]

Yamasaki H, Labar KS, Mccarthy G. Dissociable prefrontal brain systems for attention and emotion. Proc. Natl. Acad. Sci. U. S. A 2002;99:11447-11451. [PubMed: 12177452]

Zakzanis KK, Leach L, Kaplan E. On the nature and pattern of neurocognitive function in major depressive disorder. Neuropsychiatry Neuropsychol. Behav. Neurol 1998;11:111-119. [PubMed: 9742509] 


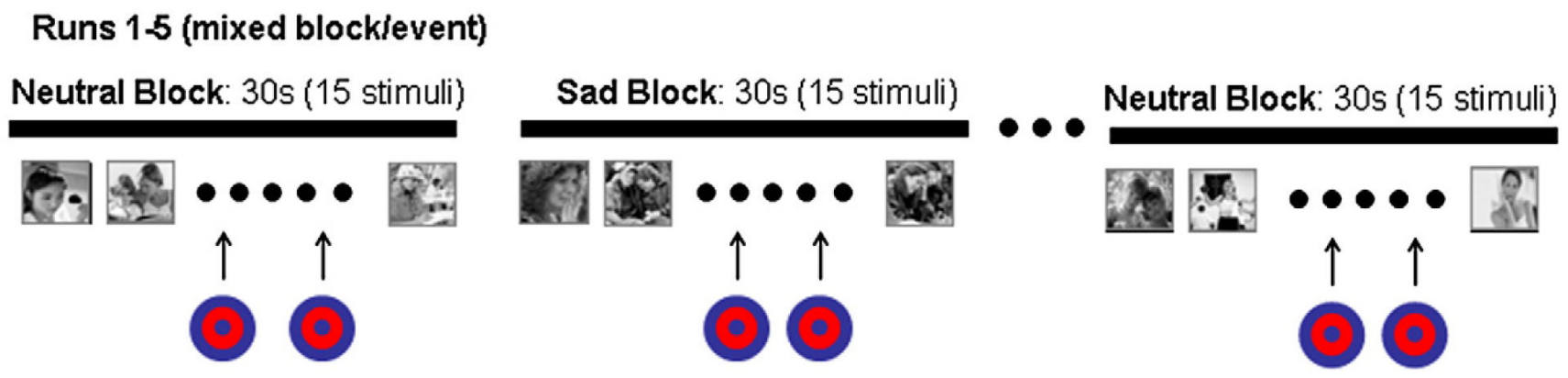

Runs 6 \& 7 (block design)

Neutral Block: 30 s (15 stimuli)

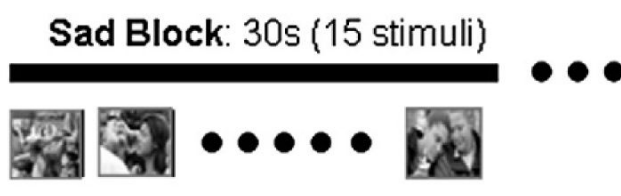

Neutral Block: 30s (15 stimuli)
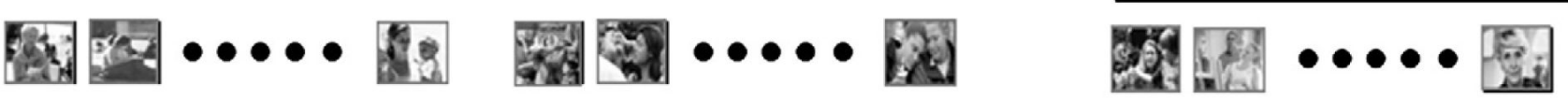

Fig. 1.

Design of the fMRI task. Runs 1-5 were a mixed block/event design with alternating 30-second blocks of neutral or sad images. Target events were presented embedded within neutral and sad blocks. Runs 6 and 7 were a passive viewing block design that presented neutral and sad blocks without target events. 


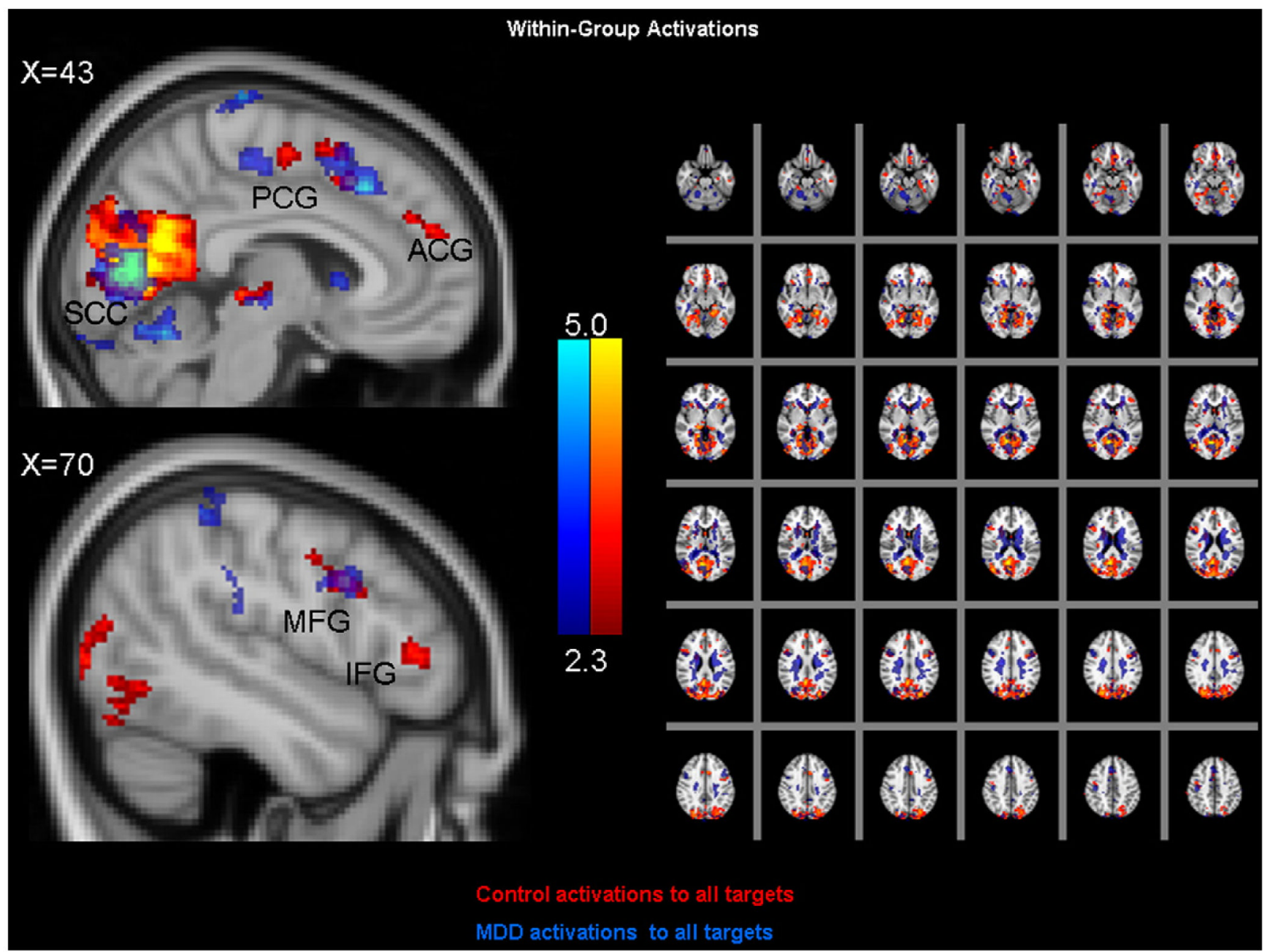

Fig. 2.

Within-group activations patterns to all target events for control (in red) and MDD (in blue) participants. Cluster mean threshold $Z>2.3$ and a cluster-corrected significance threshold of $p<0.05$. (For interpretation of the references to colour in this figure legend, the reader is referred to the web version of this article.) 


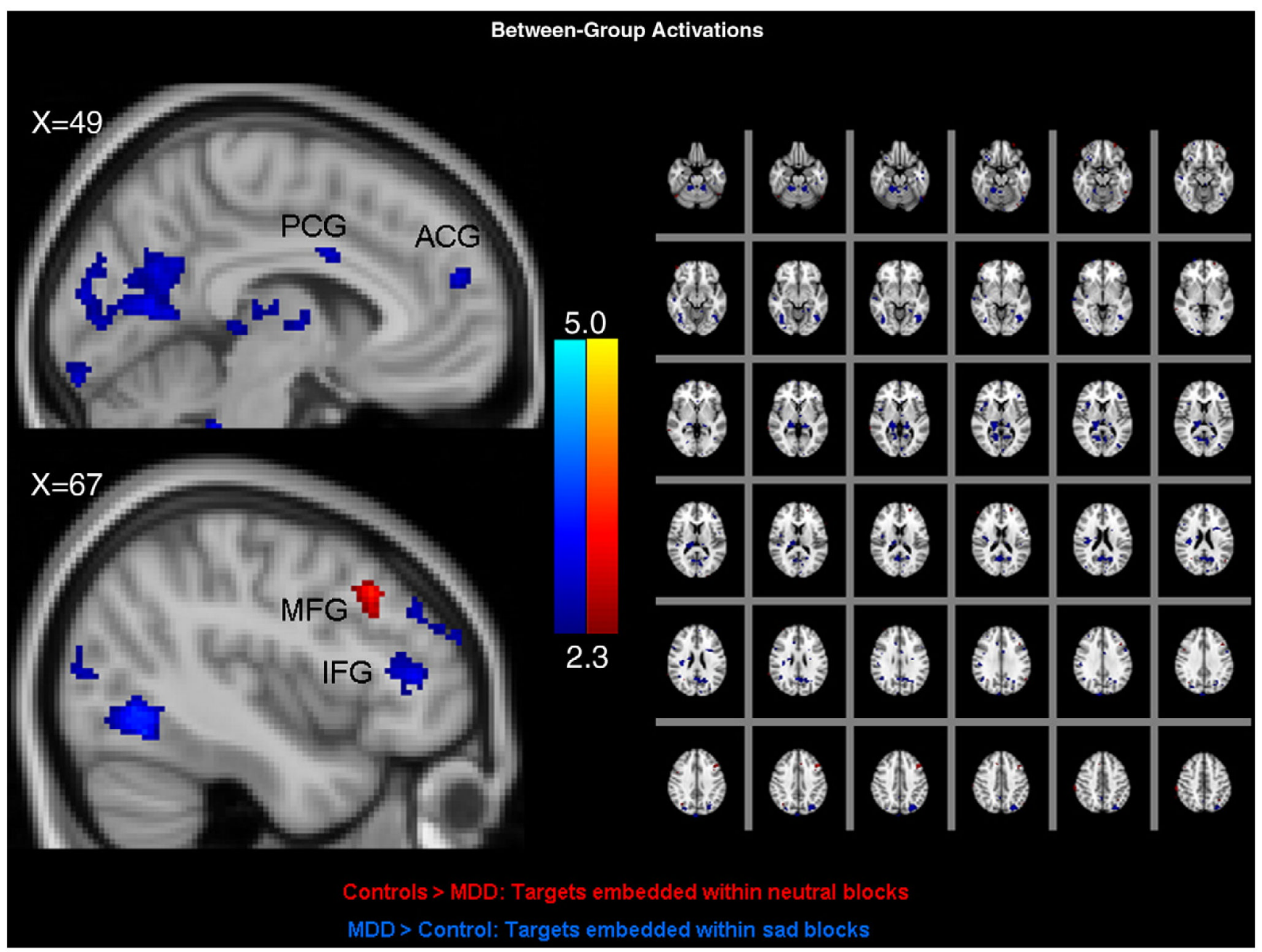

Fig. 3.

Between-group contrasts depicting brain areas with greater activation in control participants to target events embedded within blocks of neutral images (in red) and brain areas with greater activation in MDD participants to target events embedded within blocks of sad images (in blue). Note: ACG: Anterior Cingulate Gyrus; PCG: Posterior Cingulate Gyrus MFG: Middle Frontal Gyrus; IFG: Inferior Frontal Gyrus. Cluster mean threshold $Z>2.3$ and a clustercorrected significance threshold of $p<0.05$. (For interpretation of the references to colour in this figure legend, the reader is referred to the web version of this article.) 


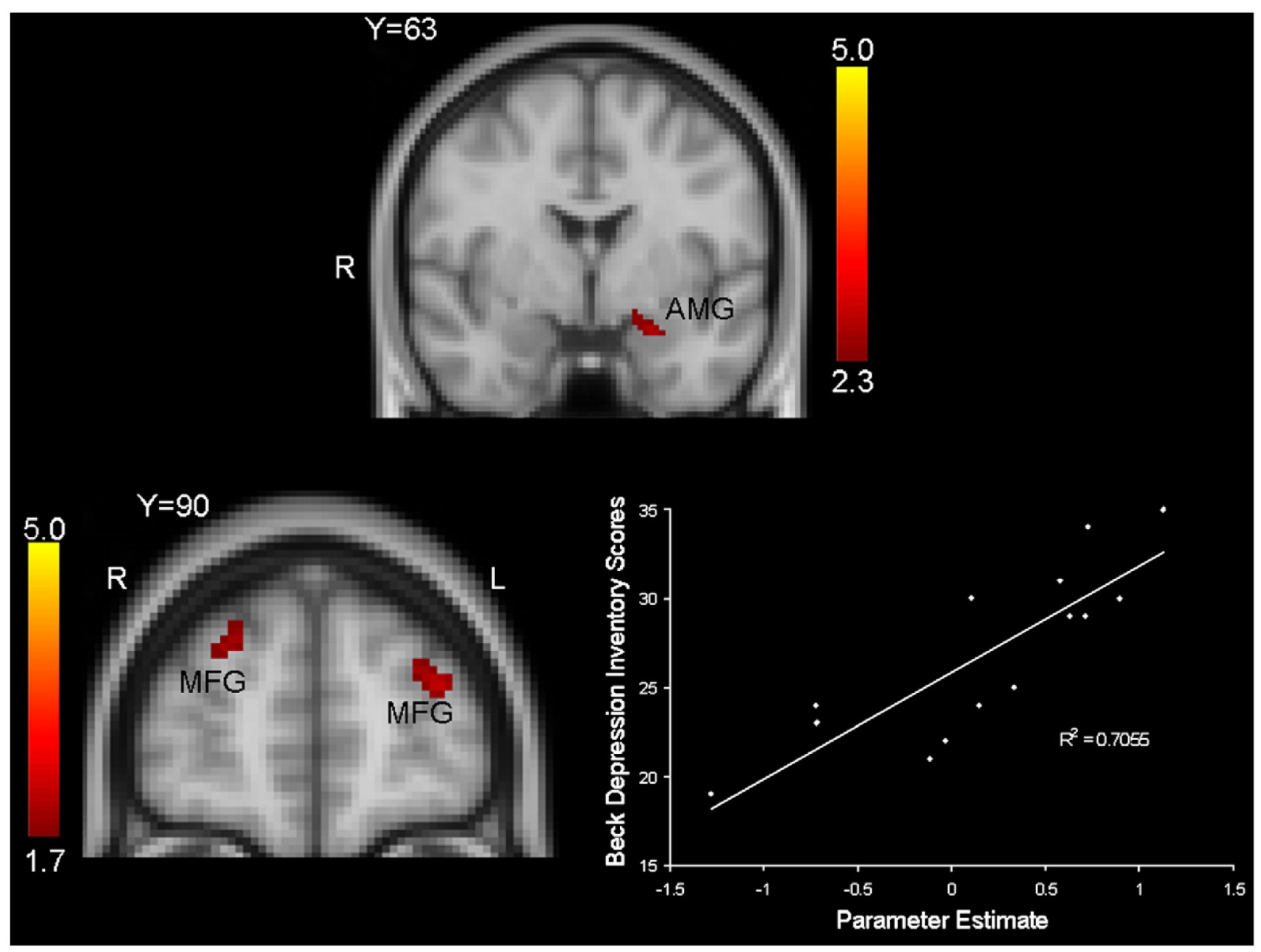

Fig. 4.

Top. Coronal view depicting relatively greater activation in the left amygdala (AMY) in MDD participants in response to block of sad images, relative to blocks of neutral images, during runs without target events (i.e., runs 6 and 7). Cluster mean threshold $Z>2.3$ and a clustercorrected significance threshold of $p<0.05$. The coordinates of peak activation in the left AMY is $32 \mathrm{~mm}, 63 \mathrm{~mm}, 25 \mathrm{~mm}$. Bottom. Coronal view depicting relations between Beck Depression Inventory (BDI) scores and midfrontal activations to target events embedded within blocks of sad images in the MDD group (left) and scatterplot of this relation (right). Note: MFG: Middle Frontal Gyrus. 

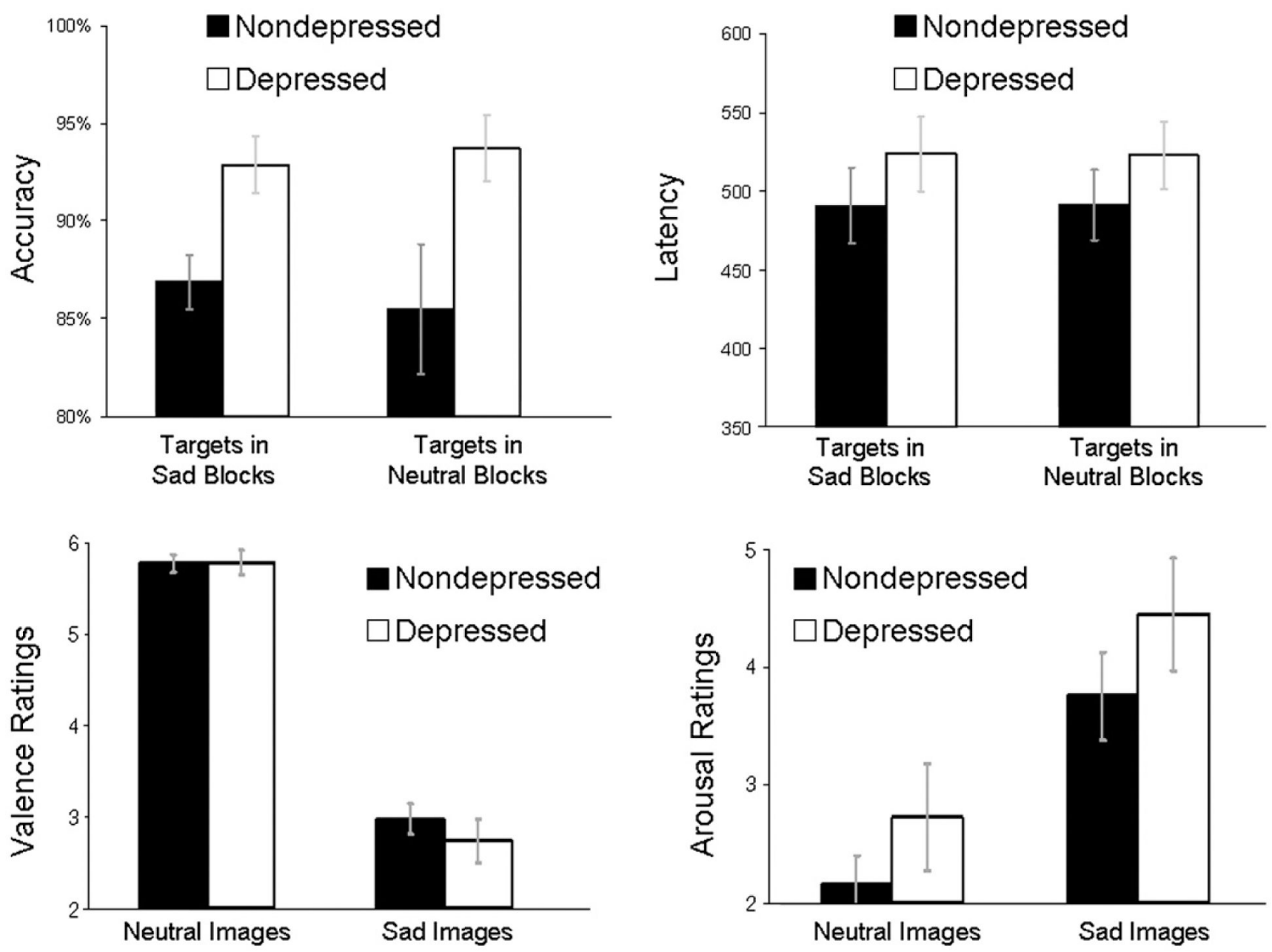

Fig. 5.

Top Left: In-scanner accuracy (percent correct) for target events presented embedded within sad and neutral blocks. Top Right: In-scanner latency (in $\mathrm{ms}$ ) for target events presented embedded within sad and neutral blocks. Bottom Left: Mean valence ratings of neutral and sad images by both diagnostic groups. The range of ratings was: 1 (extremely unpleasant) to 9 (extremely pleasant). Bottom Right: Mean arousal ratings of neutral and sad images by both diagnostic groups. The range of ratings was: 1 (not at all aroused) to 9 (extremely aroused). Note: Errors bars represent group standard errors of the mean. 


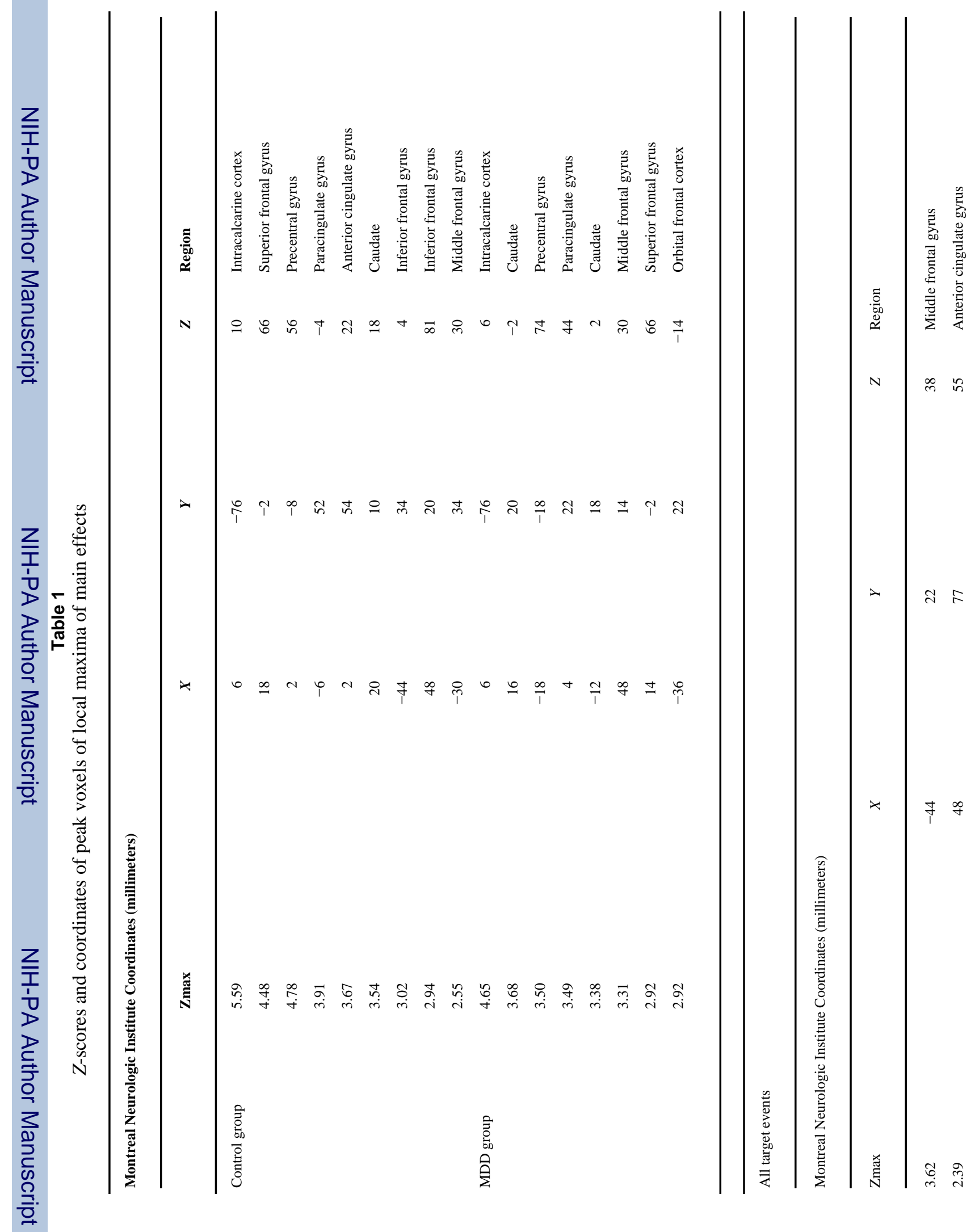




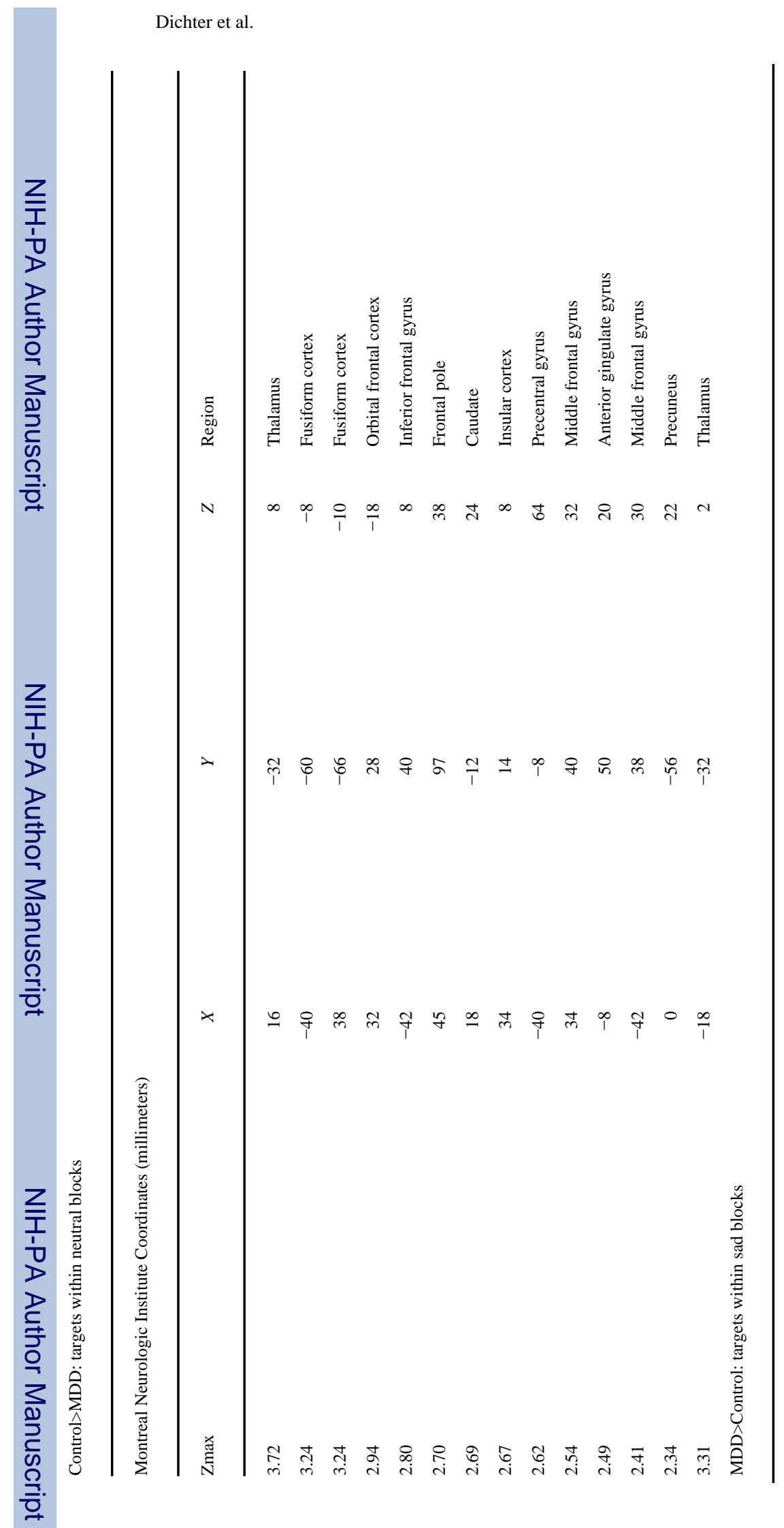

Page 19 